\title{
Diacylglycerol metabolism attenuates T-cell receptor signaling and alters thymocyte differentiation
}

\author{
M Almena ${ }^{1}$, E Andrada ${ }^{1}$, R Liebana ${ }^{1}$ and I Merida* ${ }^{* 1}$
}

Diacylglycerol (DAG) metabolism has a critical function in Ras-regulated functions in mature $T$ cells, but causal data linking defects in DAG-based signals with altered thymus development are missing. To study the effect of increased DAG metabolism in T-cell development, we engineered a membrane-targeted constitutive active version of DAG kinase- $\alpha$ (DGK $\alpha$ ). We show that transgenic expression of constitutive active DGK leads to developmental defects in T cells, with a marked accumulation of immature CD8 thymocytes and a reduction in positive selected populations. These alterations are reflected in the periphery by a CD4/CD8 cell imbalance and general T-cell lymphopenia. The results link DAG metabolism to T-cell homeostasis, and show that correctly controlled generation and consumption of this lipid at the plasma membrane ensure T-cell passage through quality-control checkpoints during differentiation.

Cell Death and Disease (2013) 4, e912; doi:10.1038/cddis.2013.396; published online 7 November 2013

Subject Category: Immunity

T cells acquire self-tolerance properties during their development, in a process that impedes generation of immunodeficiency and autoimmune pathologies. ${ }^{1}$ Common early progenitors in the thymus generate the diverse T-cell subsets: CD4, CD8, CD4 T regulatory (Treg), invariant natural killer $T$ cells and $\gamma \delta$ T cells. ${ }^{2}$ Thymocytes progress through several stages - double negative (DN), double positive (DP) and single positive (SP), depending on CD4 and CD8 coreceptor expression - while passing quality-control checkpoints. ${ }^{2}$ The first checkpoint, $\beta$-selection, is at the DN stage, where autonomous signaling by the pre-T-cell receptor (preTCR) allows thymocyte survival and proliferation, and generates clonal $\alpha \beta$ TCR diversity. ${ }^{3}$ The second control is at the DP stage, at which cells harboring mature $\alpha \beta$ TCR recognize selfantigens in the thymus cortex and medulla. Functional clones develop further (positive selection), whereas autoreactive clones are cleared from the final repertoire (negative selection). Signals triggered by the pre-TCR and the TCR not only control these two checkpoints but also generate the distinct T-cell fates. On the basis of signal strength and/or persistence, these receptors determine $\alpha \beta$ versus $\gamma \delta$ commitment at the DN stage, decide on NKT fate in preselected DP cells and regulate the specification of CD4 helper, CD8 cytotoxic and CD4 Treg populations after positive selection. ${ }^{4,5}$

TCR signaling begins with tyrosine kinase activation, which induces phospholipase $\mathrm{C}_{\gamma} 1$ (PLC $\gamma 1$ )-mediated hydrolysis of phosphatidyl-4,5-biphosphate into diacylglycerol (DAG) and inositol-1,4,5-triphosphate. One of the main DAG targets in thymocytes is RasGRP1, a Ras guanine-exchange factor (GEF) that activates the Ras/ERK pathway directly. ${ }^{6}$ DAG binding to RasGRP1 is essential for its GEF activity in vitro. ${ }^{7}$ Mice that lack RasGRP1 show defective positive selection, with severely impaired generation of SP cell populations; RasGRP1-negative thymocytes and peripheral $\mathrm{T}$ cells are unable to activate Ras/ERK pathways. ${ }^{8}$ These studies correlate with the role of Ras/ERK in selection, explain ERK sensitivity to DAG and phorbol ester treatment in T cells, ${ }^{9}$ and suggest a direct in vivo DAG function in the control of Ras/ ERK activation.

DAG kinases (DGKs) metabolize DAG by catalyzing its phosphorylation into phosphatidic acid (PA). ${ }^{10}$ DGK $\alpha$, an abundant isoform in $T$ cells, metabolizes membrane DAG after TCR stimulation, attenuating RasGRP1-mediated Ras activation. ${ }^{11}$ As a result, T cells lacking DGK $\alpha$ show enhanced ERK phosphorylation after TCR stimulation, and are resistant to induction of anergy. ${ }^{12}$ Despite its high levels in the thymus, DGK $\alpha$ deficiency causes no obvious alterations in T-cell development, probably because of redundancy with other isoforms. ${ }^{13}$ How DAG regulates the RasGRP1/ Ras/ERK pathway during development remains to be examined.

To address this question, we generated a constitutive active form of DGK $\alpha$ anchored to the plasma membrane (caDGK), which reduces local DAG levels. Using conventional T-cell models and transient caDGK overexpression experiments, we determined that alterations in DAG metabolism attenuate ERK phosphorylation, and alter the T-cell activation threshold. Transgenic expression of caDGK in the T-cell lineage allowed us to study DAG function in thymocyte differentiation. Our model defines the diverse roles of DAG during T-cell

\footnotetext{
${ }^{1}$ Department of Immunology and Oncology, Centro Nacional de Biotecnología/CSIC, 28049, Madrid, Spain

*Corresponding author: I Merida, Department of Immunology and Oncology, Centro Nacional de Biotecnología/CSIC, Darwin 3, UAM Campus de Cantoblanco, 28049 Madrid, Spain. Tel: +34 915854702; Fax: +34 913720493; E-mail: imerida@cnb.csic.es

Keywords: diacylglycerol kinase; T-cell receptor; RasGRP1; thymocyte development; signaling threshold

Abbreviations: Treg, T regulatory cells; DN, double negative; DP, double positive; SP, single positive; TCR, T-cell receptor; PLC $\gamma 1$, phospholipase C $\gamma 1$; mTOR, mammalian target of rapamacyin; DAG, diacylglycerol; PA, phosphatidic acid; GEF, guanine-exchange factor; DGK, diacylglycerol kinase; caDGK, constitutive active DGK $\alpha$; PMA, phorbol-12-myristate-13-acetate; FBS, fetal bovine serum; SEE, Staphylococcal enterotoxin E

Received 12.6.13; revised 07.8.13; accepted 05.9.13; Edited by G Raschellá
} 
development, and demonstrates the impact of these functions on peripheral T-cell homeostasis.

\section{Results}

Plasma membrane-anchored constitutive active DGK attenuates TCR signaling in T-cell lines. To generate the caDGK construct, we eliminated the negative regulatory $\mathrm{DGK} \alpha$ region $^{11}$ and fused the DGK $\alpha$ catalytic and $\mathrm{C} 1 \mathrm{~s}$ cores to the extracellular and transmembrane domains of the rat cell surface CD2 receptor (Figure 1a). This strategy has been used to generate membrane-targeted enzymes ${ }^{14}$ whose expression and localization are thus easily detected with an anti-rat CD2 antibody. Jurkat T cells transiently transfected with caDGK showed enhanced DGK activity and expressed a protein that migrated as a doublet in SDS-PAGE and western blot (Figure 1b, left). This double band indicates CD2 glycosylation, as confirmed by $\mathrm{N}$-glycosidase treatment (Figure 1b, right).

We evaluated the effect of altered DAG metabolism on TCR-triggered signals using transient overexpression experiments in the Jurkat T-cell line. Following immune synapse formation, ERK activation decreased in caDGK-expressing cells, confirming attenuation of DAG signals and extreme Ras/ERK pathway dependence on DAG levels in $\mathrm{T}$ cells ${ }^{8}$ (Figure 1c). This weaker activation did not correlate with altered phospho-ERK localization at the immune synapse, as reported, ${ }^{15}$ but with a signal decrease at the contact zone. We tested whether the caDGK-induced increase in phosphatidic acid (PA) production altered PA-delivered signals. To determine activity of the mammalian target of rapamacyin (mTOR) complex, the main PA target, ${ }^{16}$ we measured S6 phosphorylation after TCR stimulation. ERK activation after TCR stimulation was decreased in caDGK-expressing cells (Figure 1d, left) but phospho-S6 induction did not increase in caDGK cells (Figure 1d, right), suggesting that altered PA levels cause no changes. Basal and inducible phosphorylation of $\mathrm{S} 6$ protein was weaker in caDGK than in untransfected cells, which can be attributed to positive ERK control of mTOR activity. ${ }^{17}$

Analysis of $\mathrm{Ca}^{2+}$ flux in caDGK-overexpressing cells was unaltered after TCR triggering (Figure 1e), confirming selective DAG-dependent signal inhibition downstream of PLC $\gamma$ activation. Upregulation of the Ras-regulated T-cell activation marker CD69 was decreased in TCR-stimulated caDGK-overexpressing cells (Figure 1f). A more detailed
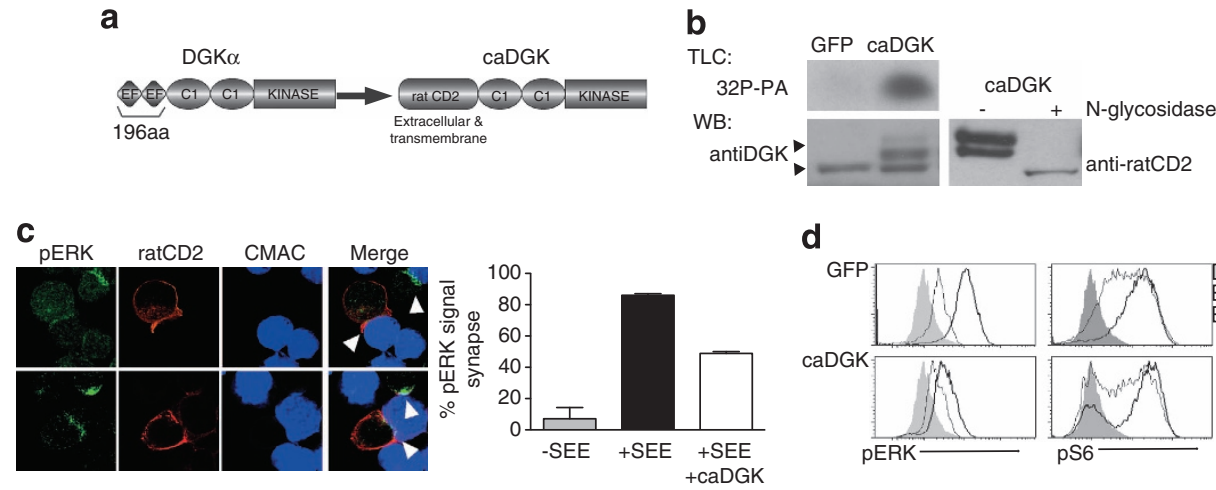

d
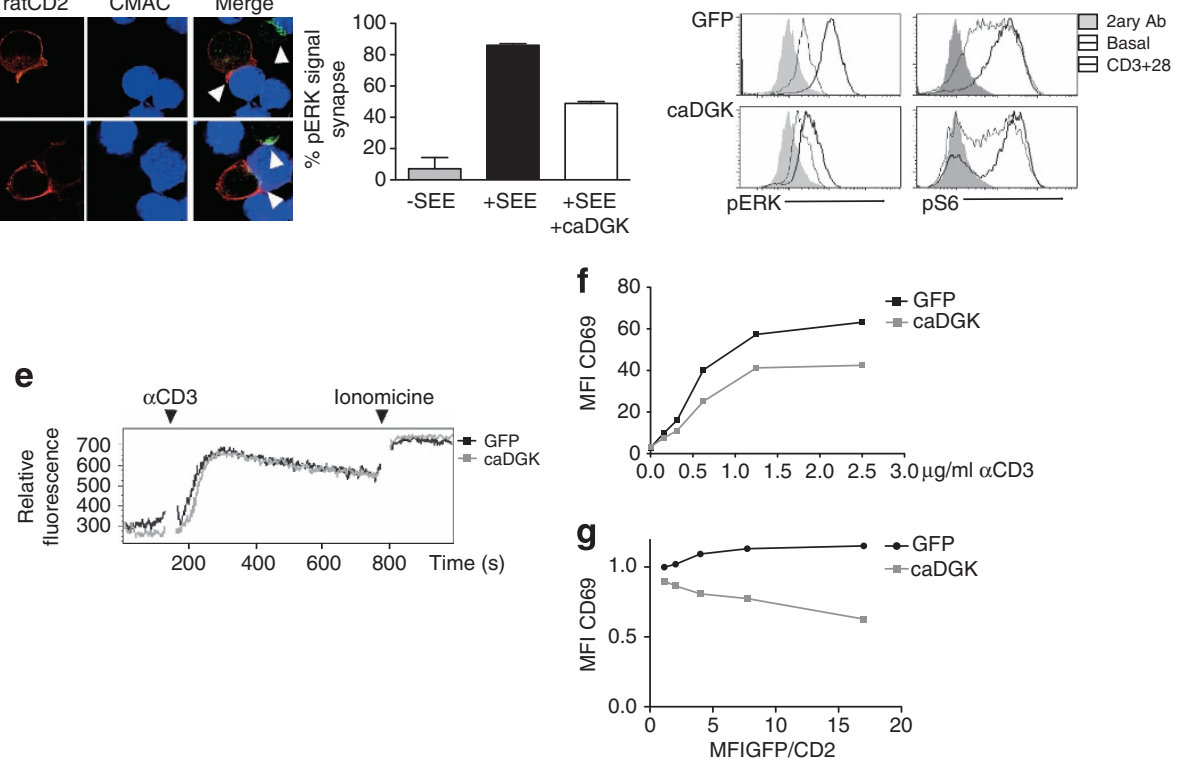

Figure 1 Constitutive active DGK generation and expression in cell lines. (a) Scheme showing caDGK construct generation. The first 196 amino acids, which encode EF-hand domains, were excised and replaced with extracellular and transmembrane domains of the rat CD2 molecule. This strategy enables caDGK localization at the plasma membrane and allows detection with an anti-rat CD2 antibody. (b) Kinase activity was determined by an in vitro reaction, caDGK expression was assessed by western blot and glycosylation by N-glycosidase treatment. (c) Immune synapses (IS) were induced between Jurkat T cells and SEE-loaded Raji cells (CMAC staining, blue). ERK phosphorylation (Alexa488, green) and caDGK expression (Cy3, red) were determined by immunofluorescence. Green-stained cells at the IS were quantified (right, mean \pm S.E.M.). (d) Jurkat cells stimulated with anti CD3 $/$ CD28 antibodies or PMA $(200 \mu \mathrm{M})$ were fixed, permeabilized and stained for phospho-ERK, phospho-S6 and rat $\mathrm{CD} 2$ expression. (e) $\mathrm{Ca}^{2+}$ flux was determined by flow cytometry in GFP- or caDGK-expressing cells after anti-CD3 $\varepsilon$ and ionomycin stimulation. (f) After $24 \mathrm{~h}$ stimulation, CD69 induction in $\mathrm{CD}^{+}$or $\mathrm{GFP}^{+}$cells was analyzed by flow cytometry (expressed as mean fluorescence intensity, MFI). (g) CD69 MFI (normalized to that of the GFP-negative population within each sample) was plotted against GFP/CD2 MFI. Representative experiments of at least three are shown in all the cases 


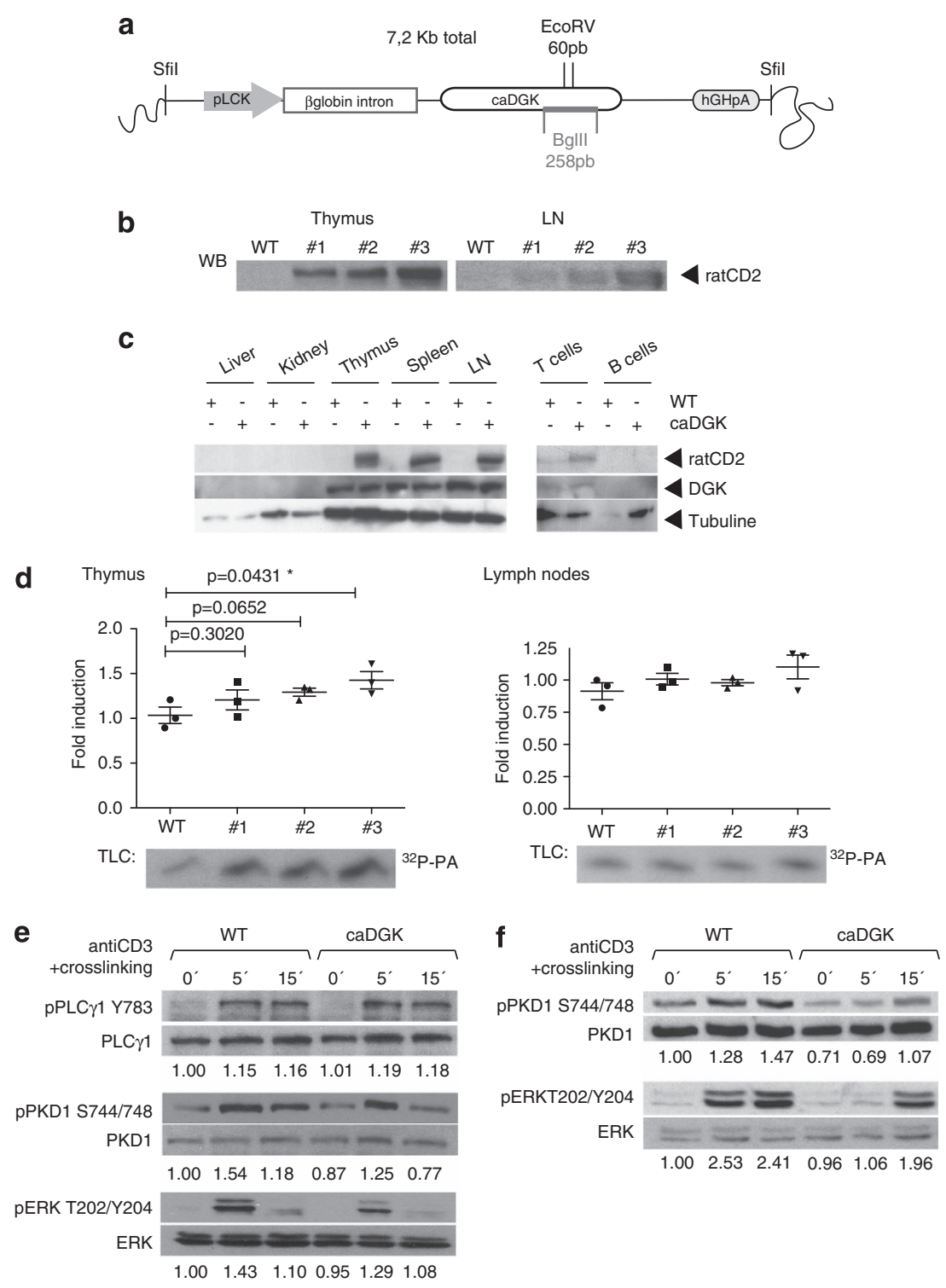

Figure 2 caDGK transgenic mice generation. (a) The caDGK construct was cloned in the p1017D vector containing the proximal Lck promoter, the $\beta$-globin intron and the human growth factor polyadenylation signal. (b) caDGK expression in founder mice was determined by western blot in total cell lysates with anti-rat CD2 antibody, in thymocytes (left) and LN cells (right). (c) caDGK expression was assesed in different tissues (left) or lymphocyte lineages (right) in cell lysates of control or transgenic mice. Anti-rat CD2 antibody was used to shown transgene expression, whereas anti-DGK $\alpha$ antibody detected endogenous enzyme. (d) In vitro kinase assay in transgenic thymocytes and peripheral T cells. Total DGK activity was measured in purified thymocyte membranes and total LN cell lysates. Data were normalized to protein loading control and $x$-fold induction represented $(n=3)$. (e) PLC $\gamma$, PKD and ERK activation after anti-CD3 $\varepsilon 2 \mu \mathrm{g} / \mathrm{ml}$ and crosslinking $5 \mu \mathrm{g} / \mathrm{ml}$ (anti-lgG antibody) stimulation was determined in thymocytes by western blot with the indicated antibodies; $x$-fold induction is quantified beneath each lane. (f) Total splenocytes of control and caDGK mice were processed as in (e) and PKD and ERK phosphorylation determined

analysis confirmed that attenuation of TCR-triggered Ras signaling was dependent on caDGK levels (Figure $1 \mathrm{~g}$ ). These experiments showed that forced TCR/PLC-mediated DAG metabolism leads to a decrease in TCR-elicited Ras/ ERK-regulated signals.

Generation of caDGK transgenic mice. Many facets of T-cell development depend on TCR signal intensity and/or persistence. Commitment to $\alpha \beta$ or $\gamma \delta$ T cell fate, as well as to the CD4 or CD8 lineage, is controlled by these mechanisms. ${ }^{4,5}$ Positive selection thresholds are also fixed by TCR signal strength, with a specific contribution by the Ras/ERK pathway. ${ }^{18}$ Given the effect of caDGK expression on TCR downstream signals and on ERK activation, we tested the consequences of abnormal DAG metabolism in vivo. To determine the functional effects of increased DGK activity in thymic development, we generated transgenic mice that expressed the caDGK protein in the T-cell lineage by subcloning the caDGK construct in the p1017D vector (Figure 2a). This vector bears the proximal Lck promoter that drives expression from the immature progenitor stages and has been used to produced mice lines with high transgene expression in thymocytes. ${ }^{19}$ Transgene insertion was detected by Southern blot (Supplementary Figure 1a) 
and three independent caDGK mouse lines were established and chosen for further study. Western blot analysis showed transgene expression in thymocytes and peripheral $\mathrm{T}$ cells in the three chosen lines (Figure $2 b$ ). Western blot analysis confirmed expression of the transgene in lymphoid organs (Figure 2c, left) as well as in specific T-cell expression (Figure 2c, right), as previously described for the promoter. Endogenous levels of DGK $\alpha$ enzyme were not altered because of caDGK (Figure 2c). Although the anti-mouse $\mathrm{DGK} \alpha$ antibody did not recognize transgene caDGK (encoded by a porcine DGK $\alpha$ ), these results demonstrated $\mathrm{DGK} \alpha$ overexpression in transgenic T cells. We determined DGK kinase activity of the three lines, and found increased total DGK activity, especially in thymocytes (Figure 2d). caDGK detection by flow cytometry was detected; although due to the decreased expression levels in cell lines, the shift of the peak was very modest, and we did not use this approach (Supplementary Figure 1b). We confirmed phenotype similarity in the three lines; experiments were performed with line no. 1 and verified in the others.

As caDGK expression was lower in transgenic mice than in cell lines, we tested whether transgene overexpression attenuated TCR signaling in primary T cells. After TCR stimulation, DAG-derived signals decreased in caDGK transgenic thymocytes (Figure 2e) and splenocytes (Figure 2f) compared with controls. Proximal TCR signaling events such as PLC $\gamma$ phosphorylation (detectable only in thymocytes) did not appear to be altered. The results indicate that, as in the case of $\mathrm{T}$ cell lines, caDGK transgene expression affects DAG-dependent signaling and early T-cell activation in primary $\mathrm{T}$ cells.
Altered phenotype in the thymus and peripheral caDGK transgenic cells. caDGK mouse thymuses were smaller and total cell numbers were $50 \%$ lower than those of wild-type (WT) mice (Figure 3a). Frequency analysis showed abnormal distribution of thymic subpopulations in caDGK mice, with increases in DN, ISP (TCR ${ }^{\text {lo }} \mathrm{HSA}^{\mathrm{med} / \mathrm{hi}}$ ) and CD8 SP cells, and a notable decrease in the percentage of DP cells (Figure $3 \mathrm{~b}$ ). Total absolute numbers reflected changes in DP and CD4 ${ }^{+}$CD8 ${ }^{\text {lo }}$ compartments (Table 1$)$.

We analyzed natural Treg (nTreg, CD4 ${ }^{+} \mathrm{CD} 25^{+} \mathrm{FoxP}^{+}$) and NKT lineages, thought to be generated by agonist selection, ${ }^{20}$ as well as $\gamma \delta$ T cells, whose number is determined by preselection signal strength. ${ }^{4}$ NKT percentages were significantly reduced in caDGK mice, and no marked alterations were observed in $\mathrm{nTreg}$ or $\gamma \delta$ T-cell populations (Figure 3c and Table 1).

We studied peripheral T-cell subset distribution in transgenic caDGK mice. Subsets in transgenic spleens tended to decrease compared with controls, although it was not significant (Figure $4 \mathrm{a}$ and Table 1). In lymph nodes (LN), which reflect the result of the differentiation process in the thymus, we observed a clear decrease in total cell numbers (Figure 4a) as well as in CD4, CD8, CD4 Treg and B-cell numbers (Table 1). CD4/CD8 T-cell ratios decreased (Figure $4 b$ ), probably due to the relative increase in thymic CD8 cell production. Transgenic peripheral blood $T$ cells showed high CD69 and CD44 expression and an altered cell surface CD62L pattern, indicating a hyperactivated phenotype for $T$ cells in these compartments (Figure 4c). These characteristics resemble those found in mice in which thymic developmental defects generate peripheral lymphopenia. ${ }^{21,22}$ a

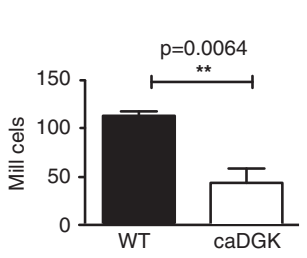

c

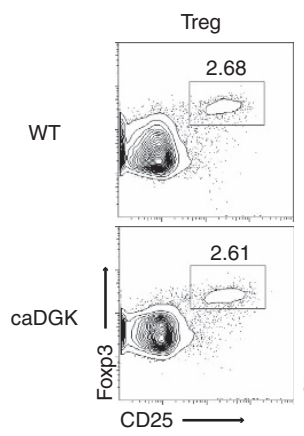

b

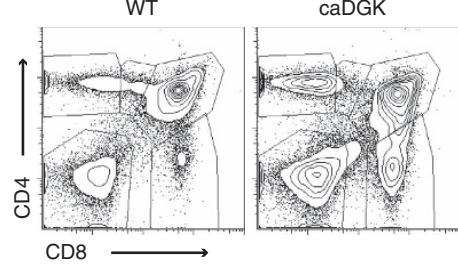

NKT

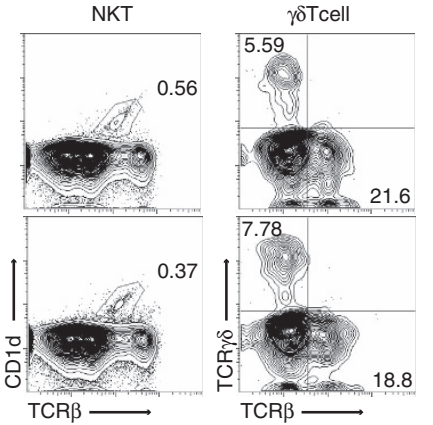

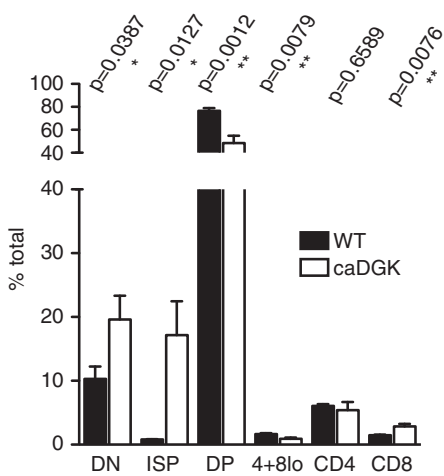

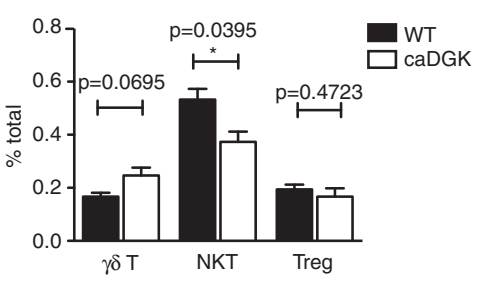

Figure 3 Altered cell numbers and populations in caDGK transgenic thymus. (a) Thymus size and cell numbers were determined in caDGK mice. (b) Subpopulations distribution of DN, DP and SP cells was analyzed by CD4 and CD8 staining and flow cytometry (left) and quantified relative to controls (right); absolute numbers are indicated in the table (bottom). (c) Flow cytometry dot plots of Treg (intracellular FoxP3 staining of the CD4SP CD25+ population), NKT (PBS-57-loaded CD1d tetramers and TCR $\beta$ staining) and $\gamma \delta$ T cells ( $\gamma \delta$ TCR and $\operatorname{CD} 3 \varepsilon$ staining). Quantification is shown on the right 
Table 1 Total absolute numbers of T-cell subsets in thymus, lymph nodes and spleen

\begin{tabular}{|c|c|c|c|c|c|c|}
\hline Thymus & DN & ISP & DP & $4+8^{10}$ & CD4 & CD8 \\
\hline $\begin{array}{l}\text { WT } \\
\text { caDGK }\end{array}$ & $\begin{array}{l}7.157 \pm 0.484 \\
6.269 \pm 1.601 \\
P=0.6748\end{array}$ & $\begin{array}{l}0.860 \pm 0.015 \\
3.732 \pm 1.643 \\
P=0.1994\end{array}$ & $\begin{array}{l}88.907 \pm 3.697 \\
24.326 \pm 11.25 \\
P=0.0020\end{array}$ & $\begin{array}{l}2.262 \pm 0.159 \\
0.619 \pm 0.395 \\
P=0.0123\end{array}$ & $\begin{array}{l}6.091 \pm 0.262 \\
2.992 \pm 1.207 \\
P=0.073\end{array}$ & $\begin{array}{l}1.900 \pm 0.044 \\
1.418 \pm 0.391 \\
P=0.3539\end{array}$ \\
\hline Thymus & & $\gamma \delta$ Tcells & & NKT & & nTreg \\
\hline $\begin{array}{l}\text { WT } \\
\text { caDGK }\end{array}$ & & $\begin{array}{l}0.1978 \pm 0.021 \\
0.2058 \pm 0.057 \\
P=0.9097\end{array}$ & & $\begin{array}{l}0.6295 \pm 0.068 \\
0.3155 \pm 0.094 \\
P=0.0353\end{array}$ & & $\begin{array}{l}0.2155 \pm 0.022 \\
0.1328 \pm 0.032 \\
P=0.0784\end{array}$ \\
\hline Spleen & \multicolumn{2}{|c|}{ CD4 } & CD8 & \multicolumn{2}{|c|}{ B cells } & Tregs \\
\hline $\begin{array}{l}\text { WT } \\
\text { caDGK }\end{array}$ & \multicolumn{2}{|c|}{$\begin{array}{l}19.14 \pm 1.591 \\
14.28 \pm 2.858 \\
P=0.1753\end{array}$} & $\begin{array}{l}10.98 \pm 1.235 \\
8.496 \pm 1.666 \\
P=0.2661\end{array}$ & \multicolumn{2}{|c|}{$\begin{array}{l}39.18 \pm 6.947 \\
30.45 \pm 8.913 \\
P=0.4515\end{array}$} & $\begin{array}{l}1.663 \pm 0.225 \\
1.245 \pm 0.333 \\
P=0.3284\end{array}$ \\
\hline LN & \multicolumn{2}{|c|}{ CD4 } & CD8 & \multicolumn{2}{|c|}{ B cells } & Tregs \\
\hline $\begin{array}{l}\text { WT } \\
\text { caDGK }\end{array}$ & \multicolumn{2}{|c|}{$\begin{array}{l}1.251 \pm 0.129 \\
0.587 \pm 0.211 \\
P=0.0404\end{array}$} & $\begin{array}{l}1.481 \pm 0.195 \\
0.557 \pm 0.020 \\
P=0.0143\end{array}$ & \multicolumn{2}{|c|}{$\begin{array}{l}0.768 \pm 0.098 \\
0.314 \pm 0.083 \\
P=0.0200\end{array}$} & $\begin{array}{l}0.214 \pm 0.010 \\
0.105 \pm 0.025 \\
P=0.009\end{array}$ \\
\hline
\end{tabular}

a

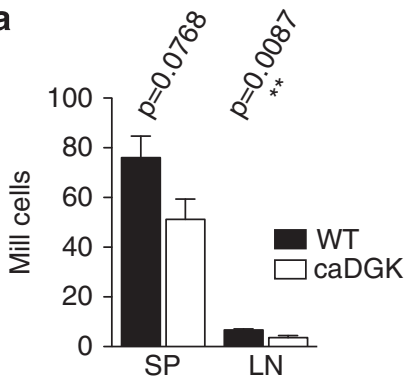

b

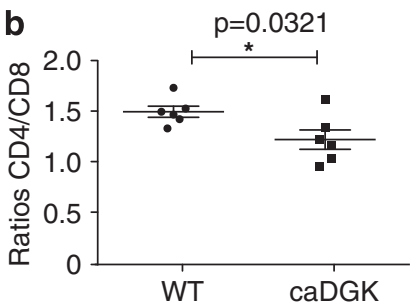

C

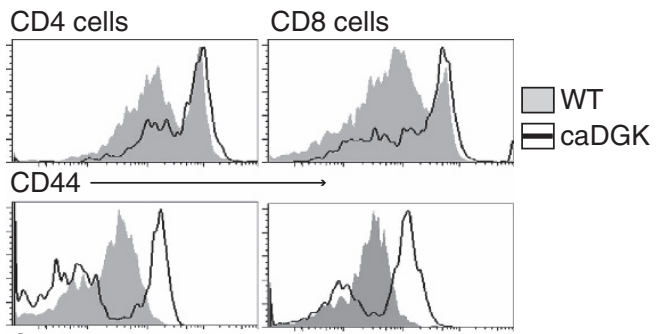

CD62L $\longrightarrow$

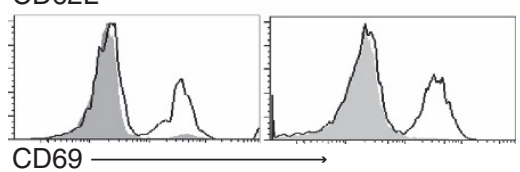

Figure 4 Peripheral T-cell populations in caDGK transgenic mice. (a) Total cell numbers of spleen and mesenteric, popliteal and axillary LNs in caDGK transgenic mice and control littermates. (b) Flow cytometry dot plots of CD44, CD69 and CD62L expression in gated peripheral blood CD4 and CD8 cells. (c) Ratios were calculated for total CD4 versus total CD8 cells in control and transgenic spleens. Results are shown for at least five independent experiments

Impaired progression of immature single positive cells in caDGK mice. Given the increased percentage of DN cells in caDGK mice, we analyzed possible developmental arrest at the $\beta$-selection checkpoint, directed by pre-TCR signaling. DN1-4 subset distribution within the DN population in caDGK mice, based on CD44 and CD25 surface marker expression, ${ }^{3}$ was similar in the percentage to controls (Supplementary Figure 2a). Analysis of intracellular TCR $\beta$-chain reorganization showed no notable differences between WT and transgenic DN cells (Supplementary Figure 2B). Moreover, cell cycle induction from DN3 to 4 stage was unaltered in caDGK mice (Supplementary Figure 2C). These results rule out a blockade during $\beta$-selection in caDGK mice.
The most pronounced defect in caDGK transgenic thymus was an accumulation of immature SP CD8 cells, a subpopulation in transition from the DN to the DP stage; they are characterized phenotypically as $\mathrm{HSA}^{\text {high }} \mathrm{TCR}^{\text {low }} \mathrm{CD} 69^{\text {med/low }}$, and upregulate and express the CD8 coreceptor before CD4. ${ }^{23}$ To confirm this arrest, we analyzed fetal thymus at embryonic day (E)18.5, when populations are distributed between DN, ISP and DP, such that the only $\mathrm{CD}^{+}$cells are ISP. ${ }^{24}$ caDGK mouse fetal thymus showed a marked decrease in cell number (Figure 5a), caused by ISP cell accumulation; percentages and total number of DP cells were therefore lower (Figure 5b). This finding indicates that lower cell numbers in fetal and adult thymuses were due to impaired 
a

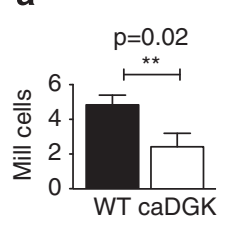

b

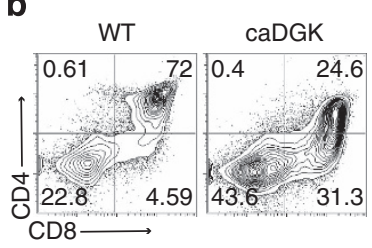

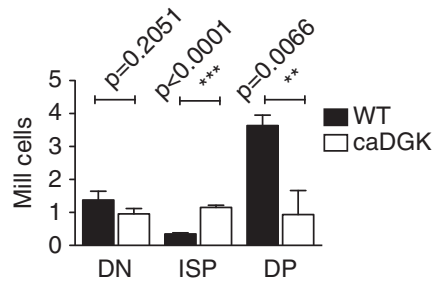

c

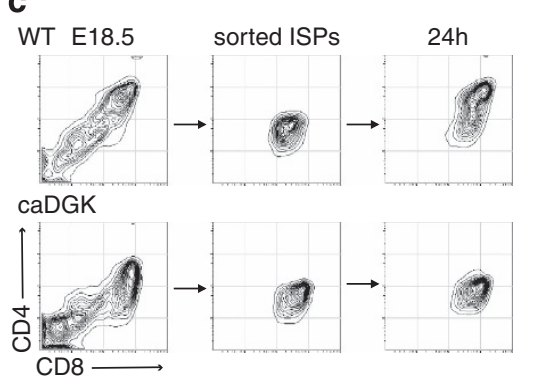

d

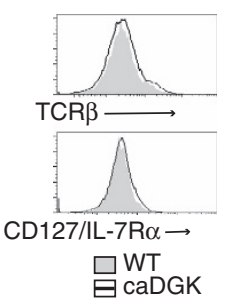

e

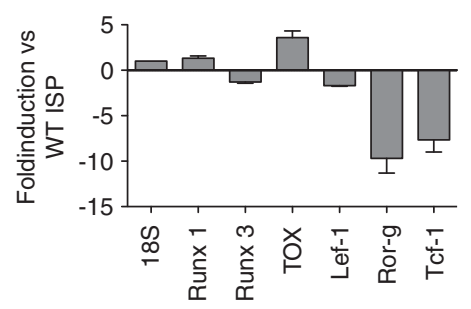

g

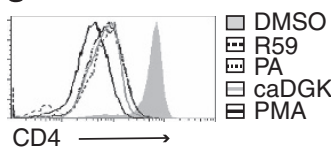

f

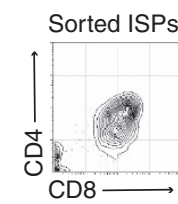

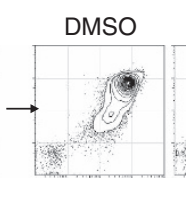

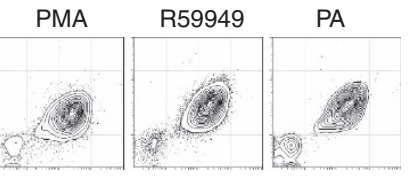

CD4

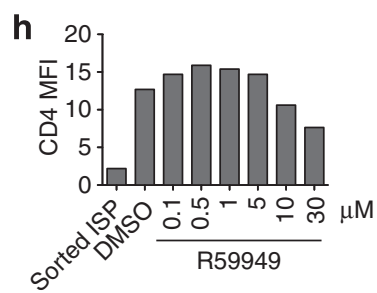

Figure 5 Characterization of ISP subpopulation in caDGK transgenic mice. Cell numbers were determined in E18.5 fetal thymus from control and caDGK transgenic mice (a) and subpopulations analyzed (b). (c) ISP CD8 ${ }^{+} \mathrm{CD} 4{ }^{-}$cells from E18.5 fetal thymus were isolated by fluorescence-activated cell sorter and cultured in RPMI. After $24 \mathrm{~h}$, cells were collected, stained for CD4 and CD8 surface markers and analyzed by flow cytometry. (d) Overlays of TCR $\beta$ and CD127 surface expression in gated $\mathrm{CD}^{-}{ }^{-} \mathrm{CD} 8^{+} \mathrm{HSA}^{\mathrm{lo} / \mathrm{med}}$ ISP population in control and caDGK mice. (e) mRNA levels of the indicated molecules were analyzed by real-time qRT-PCR of sorted CD4 ${ }^{-} \mathrm{CD} 8^{+}$ ISP thymocytes; expression was normalized to the $18 \mathrm{~S}$ housekeeping gene in the same samples. (f) ISP cells were cultured in the presence of PMA (200 $\mu \mathrm{M}$ ), R59949 $(30 \mu \mathrm{M})$ or PA vesicles $(100 \mu \mathrm{M})$ and analyzed for CD4 and CD8 expression $24 \mathrm{~h}$ later. (g) Overlay of CD4 expression in the assayed conditions is shown. (h) ISP cells were isolated and culture as in (c) and CD4 upregulation was determined in the presence of different concentrations of the DGK inhibitor

ISP-to-DP progression. We then examined differentiation capacity by isolating the ISP population and culturing these cells in vitro. Whereas control cells showed upregulated CD4 levels and became DP cells, ${ }^{25}$ transgenic ISP cells remained $\mathrm{CD}^{+}{ }^{+} \mathrm{CD} 4^{10}$ (Figure $5 \mathrm{C}$ ). Analysis of ISP progression parameters $^{25,26}$ showed normal TCR and IL-7R $\alpha$ /CD127 levels (Figure $5 \mathrm{~d}$ ) but a moderate decrease in the expression of LEF-1, TCF and ROR $\gamma$ transcription factors (Figure 5e), essential for ISP conversion to DP. This shows that enhanced DGK expression in early T-cell progenitors results in diminished expression of genes required to promote ISP-to-DP differentiation.

The ISP blockade in caDGK mice suggests that differentiation through this stage requires adequate DGK-mediated homeostasis of DAG and PA levels. We examined the effect of altering those lipids on the ISP-to-DP transition of WT cells. Increasing DAG levels by pharmacological DGK inhibition or mimicking a DAG increase by treatment with phorbol-12-myristate-13-acetate (PMA) impaired IS-to-DP conversion (Figure 5f). PA addition to cultured ISP cells also prevented differentiation (Figure 5f). The blockade to DP transition, determined as the capacity to upregulate CD4, was therefore similar after transgenic caDGK overexpression, DGK inhibition or PA treatment, and was more pronounced when PMA was added to the culture (Figure $5 \mathrm{~g}$ ). We confirmed the specificity of the effect observed with R59949 inhibitor in a dose-response assay (Figure $5 \mathrm{~h}$ ); viability of ISP thymocytes did not seem to be affected by DGK inhibition (not shown), contrary to the described DP sensitivity to this treatment. ${ }^{27}$ These results show the need for precise control of DAG and PA signaling for ISP progression, and confirm an essential DGK function in ISP cell transition to the DP stage.

Defective SP differentiation in caDGK transgenic mice. In addition to the partial arrest at the ISP stage, the altered phenotype beyond the DP stage suggested selection defects 
a
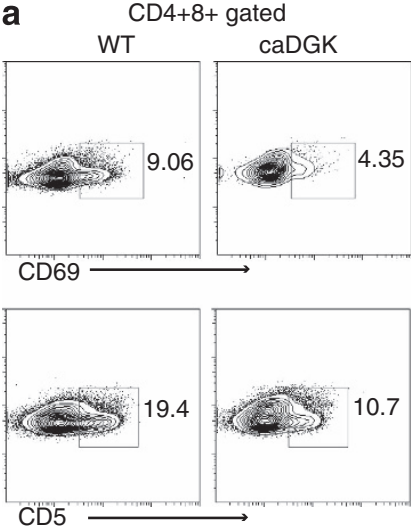
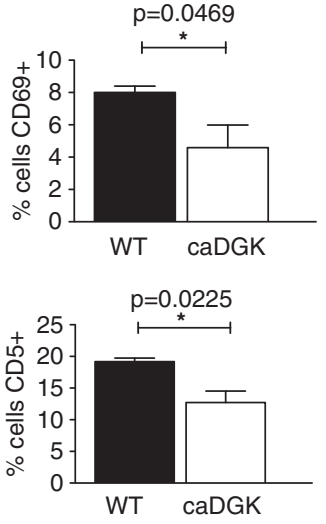

b

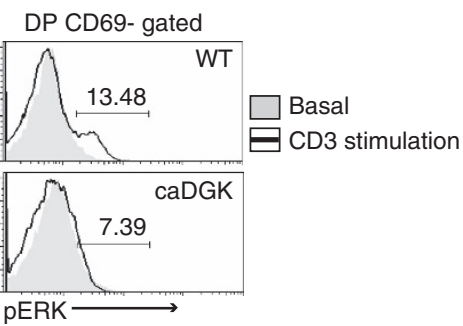

C

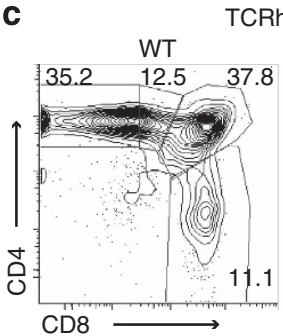

TCRhi gated

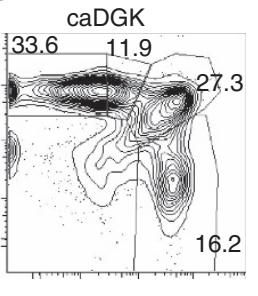

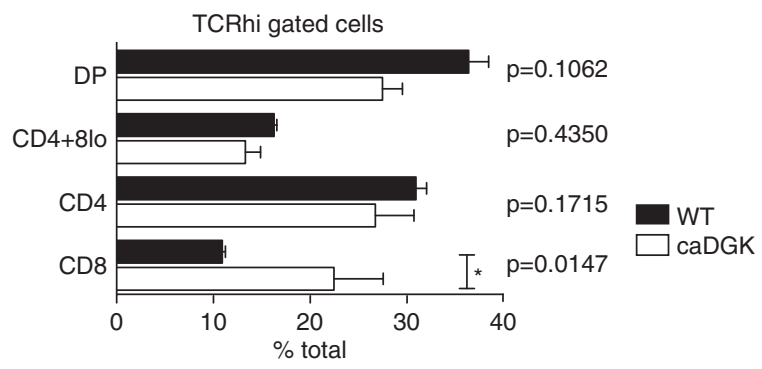

TCRhi gated cells d

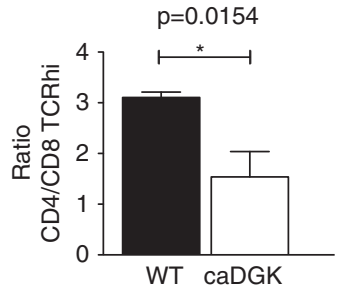

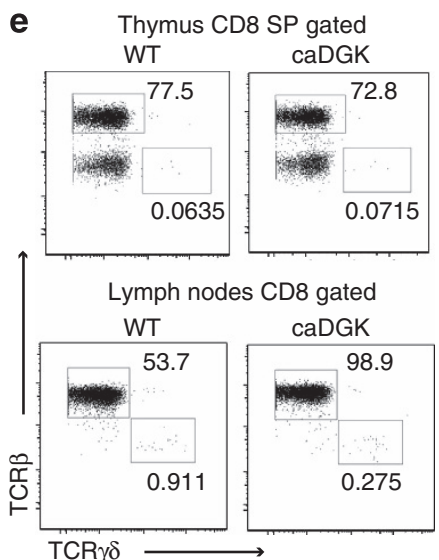

CD8 $\gamma \delta$ T cells

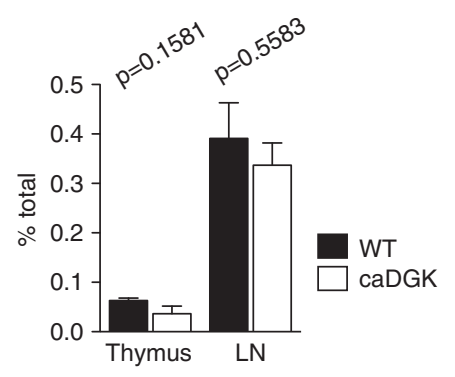

Figure 6 Defective positive selection in caDGK transgenic mice. (a) Cell surface CD69 and CD5 expression was analyzed in gated DP subpopulations. Quantification is shown on the right. (b) Intracellular phospho-ERK staining after anti-CD3 stimulation, assessed by flow cytometry in gated, preselected DP CD69 ${ }^{-}$thymocytes. (c) Dot plots of CD4 versus CD8 profiles in gated TCR $\beta^{\text {hi }}$-expressing cells. Quantification is shown on the right. (d) Comparative ratios of CD4 SP TCR ${ }^{\text {hi }} / C^{2} 8$ SP TCR ${ }^{\text {hi }}$ cells. (e) TCR $\beta$ and $\mathrm{TCR} \gamma \delta$ surface expression is analyzed in gated CD8 SP thymocytes and in LN CD8 T cells. Quantification is shown on the right

in caDGK mice. We analyzed surface expression of CD69 and CD5, whose upregulation in DP cells is a hallmark of a positive selection. ${ }^{28}$ Cell surface expression of CD69, which is upregulated by Ras activation, was lower in DP transgenic cells (Figure 6a, top). Cell surface CD5 expression, usually interpreted as a measure of TCR signal strength, ${ }^{29}$ was also lower in caDGK transgenic DP cells than in those of controls (Figure 6a, bottom). ERK activation, a critical positive selection signal in preselected DP $\mathrm{CD}^{-}{ }^{-}$cells, was low in transgenic caDGK thymocytes (Figure 6b). Analysis of selected populations expressing high TCR levels showed an imbalance toward the CD8 lineage, with CD4/CD8 ratios decreased by $\sim 50 \%$ in caDGK mice (Figure $6 \mathrm{c}$ ); this resembled the ratios found in peripheral T cells (Figure $4 \mathrm{~b}$ ).
The increase in the CD8 compartment corresponds to CD8 $\gamma \delta$ cells in RasGRP1 ${ }^{-/-}$mice; ${ }^{30}$ thymic CD8 SP and peripheral CD8 cells were analyzed for TCR $\gamma \delta$ expression, without finding significant differences (Figure 6e). The results indicate that caDGK expression beyond the DP stage generates selection defects and altered SP thymocyte progression.

\section{Discussion}

DAG is a lipid second messenger of particular relevance in lymphocyte biology; it accumulates at the T-cell immune synapse during antigen presentation, where it regulates proteins with functions crucial for T-cell activation, 
proliferation and differentiation. ${ }^{31}$ We engineered the caDGK chimera protein to study the DAG contribution to TCR signaling independent of tyrosine kinase activation or $\mathrm{Ca}^{2+}$ input. Transgenic mice expressing this construct under the control of the early Lck promoter allowed us to assess the in vivo effect of DAG depletion in T-cell development. In this process, the role of DAG has remained largely elusive, in spite of the essential functions ascribed to the DAG-regulated molecule RasGRP1. ${ }^{8}$ We demonstrate that attenuation of DAG-regulated signals alters two developmental steps: the transition from the ISP-to-the DP stage, and the selection of SP cells from DP populations.

Experiments in T-cell lines showed that caDGK overexpression leads to general attenuation of TCR signaling as a direct result of decreased RasGRP1/Ras/ERK pathway activation. Signal intensity, measured as CD69 induction, was weaker in caDGK-expressing cells than in controls. These results confirm DGK ability to act as a brake on T-cell activation $^{12,32}$ and support studies describing feedback loops by which the Ras/ERK pathway regulates upstream molecules such as Lck. ${ }^{33}$ caDGK overexpression not only causes accelerated DAG metabolism but also increases PA levels. In non-lymphoid systems, PA binds to Raf-1, Sos and mTOR, activating the Ras/ERK and mTOR/S6 kinase axes. ${ }^{16}$ We monitored these pathways in caDGK-expressing T cells, and observed decreased Ras/ERK and mTOR/S6 induction, which differ from the positive PA function in other systems. The data show that the alterations caused by caDGK overexpression in T cells are due mainly to decreased DAG signaling rather than to enhanced PA production.

caDGK transgene expression in the murine T-cell lineage leads to clear differentiation defects. Attenuated DAG signaling in transgenic DP thymocytes reduces ERK activation and CD69 induction, both indicators of positive selection. Mature T-cell populations are consequently skewed toward the CD8 lineage, suggesting that CD4 SP cells are selected inefficiently or at a lower frequency than CD8 SP cells. DAG generation must thus be sustained to maintain the persistent TCR (and especially ERK) signaling necessary for CD4 SP selection. This explains the deviation toward the CD8 lineage, as loss of ERK function increases the CD8 SP population. ${ }^{34}$ In close correlation with its dependence on RasGRP1 function, ${ }^{35}$ NKT cell generation is low in caDGK transgenic mice.

The phenotype of RasGRP1 ${ }^{-1-}$ has demonstrated that this GEF for Ras is essential for positive thymic selection. Our data from caDGK transgenic indicate the need of adequate DAG generation during positive selection of thymic subpopulations. Certain similarities are shared between caDGK, PLC $\gamma 1^{-1-}$ conditional $^{21}$ and Itk $^{-1-}$ mice. ${ }^{36}$ In all cases, there is positive selection failure and general T-cell lymphopenia. As Itk is the kinase that activates PLC, decreased DAG levels are expected in all three models, due either to defective lipid production $\left(\mathrm{PLC}^{-/-}\right.$, $\mathrm{Itk}^{-/-}$) or to lipid enhanced metabolism (caDGK transgenics). TCR signal strength, measured as CD5 expression, is decreased in the three models, and $\mathrm{Itk}^{-1-}$ and caDGK mice have fewer NKT cells than WT mice. These shared characteristics define DAG functions during selection, and confirm the control of PLC and DGK activities in RasGRP1/ERK axis activation.
The similar developmental regulation of their expression implies coordinated RasGRP1/DGK $\alpha$ functions; both proteins are upregulated after $\beta$-selection, and reach maximum expression in DP thymocytes ${ }^{27,37}$ (Immunological Genome Project data bank; http://www.immgen.org/). DP cells need graded, low-intensity signals for selection; slight changes in DAG levels at the plasma membrane can easily generate changes in ERK activation. This interplay between DGK $\alpha$ and RasGRP1 was also shown during anergy induction, ${ }^{12,38}$ another weak signaling situation in which ERK induction is attenuated, but had not been clarified for T-cell development. When strong activation of the DAG/RasGRP1/Ras axis is needed, as in the case of mature T-cell expansion, DGK $\alpha$ is downregulated at the transcriptional level. ${ }^{39}$

Our results show new facets of DGK function during thymic selection, and complement previous studies of DGK-deficient mice. ${ }^{13} \mathrm{DGK} \alpha^{-1-}$ or $\zeta^{-1-}$ deficient mice show no developmental defects, suggesting redundancy of these isoforms, a fact corroborated by the blockade at the DP stage of $\mathrm{DGK} \alpha^{-1-} \zeta^{-1-}$ mice. The SP generation defect in these mice can be partially rescued by exogenous PA addition, suggesting that DGK-dependent survival or mitogenic signals at the DP stage are dependent on PA functions. The requirement for DGK-mediated $P A$ generation for adequate DP thymocytes development is in accordance with previous data showing survival defects in response to pharmacological DGK inhibition in the DP populations. ${ }^{27}$ These experimental approaches suggest that an adequate balance of DAG and PA signals is required for DP-to-SP progression, but the abrupt changes in lipid levels in both models makes it difficult to assess their exact contribution to selection events. Our experimental gain-of-function approach, with milder changes in DGK activity, will be of help for additional evaluation of the input of DAG metabolism to those mechanisms by which signal intensity affects T-cell selection.

Transgenic mice expressing caDGK show a partial blockade at the ISP stage, during the DN-to-DP thymocyte transition. This is probably a consequence of overexpressed DGK activity in early T precursors that express very low levels of endogenous DGK $\alpha .{ }^{27}$ Inhibitor experiments with embryonic ISP cells from WT mice suggest a strict dependence on DGK function, despite low DGK $\alpha$ expression at this stage (http: //www.immgen.org/). Conversion to DP thymocytes requires a precise equilibrium of signals. Positive signals from the pre-TCR are needed for DN-to-DP transition; nonetheless, induction must be downregulated for some factors (such as IL-7R $\alpha, \beta$-catenin or Egr) to allow ISP-to-DP transition. ${ }^{40}$ Preselected DP thymocytes must be preactivated, which increases the signaling threshold and permits interpretation of low-intensity signals during selection. ${ }^{41,42}$ Our findings suggest that DGK activity contribute to the regulation of DAG levels at the ISP stage, such that slight alterations provoke a developmental blockade. Like the caDGK mice, ISP cells also accumulate in class I- and II-restricted RasGRP1 ${ }^{-1-}$ mice, where they resemble immature cells but have cytolytic potential. ${ }^{43}$ Although the molecular mechanisms of ISP biology are not well understood, our data indicate that an additional checkpoint is established at this point in the development to guarantee quality of the DP subpopulation. 
In summary, we demonstrate that enhanced DAG metabolism attenuates TCR signaling, altering positive selection thresholds and the fate of selected $T$ cells. This leads to a marked effect on the two main TCR-dependent developmental checkpoints, ISP-to-DP transition and DP-to$\mathrm{SP}$ selection. The mechanism used by $\mathrm{T}$ lymphocytes, by which DAG membrane levels are translated into intensity and duration of Ras/ERK-mediated signals, could provide the extraordinary plasticity needed for selection and maturation of the T-cell repertoire. Expression of the DAG effector (RasGRP1) and the DAG-metabolizing enzymes (DGK) would help to establish developmental thresholds for homeostatic activation of Ras signals during DN-to-DP transition, and could provide a means to control TCR signal intensity and duration in the DP population. The contribution of RasGRP1 to human diseases, including autoimmune alterations and leukemia, suggests the need for better understanding of the DAG contribution to T-cell regulation and function.

\section{Materials and Methods}

Reagents. Orthovanadate, phenylmethylsulfonyl fluoride (PMSF), poly-DLlysine, paraformaldehyde (PFA), PMA, Igepal CA-630 (NP40), Triton X-100 and fetal bovine serum (FBS) were purchased from Sigma (St. Louis, MO, USA) and R59949 was obtained from Calbiochem (Darmstadt, Germany). Leupeptin, aprotinin and N-glycosidase were from Roche (Indianapolis, IN, USA), RPMI-1640 and L-glutamine from BioWhittaker (St. Louis, MO, USA). Staphylococcal enterotoxin E (SEE) was from Toxin Technology (Sarasota, FL, USA). Horseradish peroxidase (HRP)-conjugated goat anti-mouse and -rabbit IgG were from Dako (Carpinteria, CA, USA). Lipids for DGK assays were from Avanti Polar Lipids (Alabaster, AL, USA). CellTracker Blue CMAC (7-amino-4-chloromethylcoumarin), BODIPY 630/650 succinimidyl ester and Fluo-3 AM fluoropure grade were from Molecular Probes (Grand Island, NY, USA).

Plasmids and constructs. For the pEFbos caDGK construct, the indicated primers (Supplementary Table 2) were used to generate Notl and Sall sites by directed mutagenesis (QuickChange Site-Directed Mutagenesis Kit; Stratagene, La Jolla, CA, USA) in pEFbos EGFPDGK $\alpha \Delta 196 .^{11}$ The Notl/Sall restriction product was subcloned into pEFBos $\mathrm{rCD}_{2} 110,{ }^{44}$ replacing p110 cDNA. The p1017D vector ${ }^{19}$ was kindly donated by Dr RJ Kay (University of British Columbia, Vancouver, BC, Canada). This is a vector derived for the Ick proximal promoter vector 1017 that directs transgene expression exclusively in T cells. For p1017D caDGK, we used site-directed mutagenesis to generate Mlul sites in pEFbos caDGK (primers used were: $5^{\prime}$-CCTTCAAGGCGATGGTGGAATTGCGGC CGCTGTTCC-3' (Notl site) and $5^{\prime}$-CCTCTAGATGCATGGTCGACGCGGCCAAT T-3' (Sall site)) and p1017D (5'-GGAGACCCAACCTTGGTACGCGTTCGGATCC AAGATGAGG-3' (Mlul site)). The Mlul/Sall restriction product was subcloned into p1017D. Primers used for genotyping were: 5'-AGATCACCCACAGGAACCAG-3'; 5'-CTCGACCTTGTCCATGTCCT-3'.

Mice. Mice were housed in specific pathogen-free conditions and handled in accordance with Spanish and European directives. Experiments were approved by the Ethics Committee of the Centro Nacional de Biotecnologia (CSIC). C57BL/6J mice were from Jackson Laboratories (Bar Harbor, ME, USA). Transgenic caDGK mice were generated by pronuclear microinjection of a linear Sfil-digested p1017D-caDGK fragment in C57BL/6J oocytes.

Statistics. In all figures, values are given for mean \pm S.E.M. Statistical analysis was performed using a non-paired two-tailed t-test $(n=5 ; P<0.05)$ with GraphPad Prism software (La Jolla, CA, USA).

Southern blot. Genomic DNA $(10 \mu \mathrm{g})$ was digested with EcoRV enzyme, separated by DNA electrophoresis and transferred to $\mathrm{N}^{+}$nylon membrane (Amersham, Uppsala, Sweden). A specific probe was obtained by Bgll digestion of transgene caDGK $c D N A$ and $\left[\alpha{ }^{32} \mathrm{P}\right] \mathrm{dCTP}$-labeled (Amersham Rediprime Random Prime Labeling, Little Chalfont, UK); the membrane was hybridized with ExpressHyb (Clontech, Mountain View, CA, USA).
Cell lines and transient transfection. Jurkat and Raji cells were cultured in RPMI supplemented with $10 \%$ FBS, $2 \mathrm{mM}$ glutamine and $10 \mathrm{mM}$ HEPES. Jurkat cells in exponential growth were electroporated (20 $\mu \mathrm{g}$ DNA, 270 V $975 \mu \mathrm{F})$ in a Gene Pulser (Bio-Rad, Berkeley, CA, USA), transferred to complete medium and assayed after $24 \mathrm{~h}$

PA preparation. Vesicles were prepared by resuspending PA in $150 \mathrm{mM}$ $\mathrm{NaCl}-10 \mathrm{mM}$ Tris- $\mathrm{HCl}, \mathrm{pH} 8.0$, and sonicated $(10 \mathrm{~min})$. The suspension was added to the culture to a final concentration of $100 \mu \mathrm{M}$. Given the short half-life of PA, this process was repeated three times during the treatment period.

Western blot. Cells were suspended in lysis buffer $(150 \mathrm{mM} \mathrm{NaCl}, 10 \mathrm{mM}$ $\mathrm{NaF}, 10 \mathrm{mM} \mathrm{Na} \mathrm{P}_{2} \mathrm{O}_{7}, 50 \mathrm{mM}$ Tris- $\mathrm{HCl}, \mathrm{pH} 7.4,1 \% \mathrm{NP} 40,1 \mathrm{mM}$ orthovanadate, $1 \mathrm{mM}$ PMSF and $10 \mu \mathrm{g} / \mathrm{ml}$ each aprotinin and leupeptin), incubated on ice and centrifuged. Supernatants were assayed for total protein (DC Protein Assay; Bio-Rad) and equivalent protein amounts analyzed by SDS-PAGE, transferred to nitrocellulose membranes (Amersham) and blotted with the indicated primary antibodies. The antibodies for western blot analysis anti-p(Ser)PKC substrate; antipPKD S744/748; anti-plkB $\alpha$ S32/36; anti-pPLC $\gamma$ Y783; pZAP70 Y319; anti-pLAT Y171; anti-LAT; anti-pERK T402/Y404; anti-ERK; anti-pS6 S235/236 and anti- $\beta$ actin were purchased from Cell Signaling (Beverly, MA, USA). Anti-PLC $\gamma$ (B-6-4) was from Millipore (Billerica, MA, USA); anti-ZAP70 was from BD Transduction Lab (Franklin Lakes, NJ, USA). Anti-human DGK $\alpha$ was a generous gift of Dr. WJ van Blitterswijk (Netherlands Cancer Institute, Amsterdam, The Netherlands). Antimouse DGK $\alpha$ was raised against a peptide recognizing amino acids 602-615 in the rat sequence. HRP-conjugated anti-rat or -mouse secondary antibodies and ECL (Amersham) were used for signal detection.

In vitro kinase assay. Cells were lysed by sonication in DGK assay buffer (50 mM Tris- $\mathrm{HCl}, \mathrm{pH} 7.4,100 \mathrm{mM} \mathrm{NaCl}, 0.25 \mathrm{mM}$ saccharose, $1 \mathrm{mM}$ DTT, $1 \mathrm{mM}$ EDTA, $5 \mathrm{mM}$ EGTA, and the protease inhibitors PMSF, leupeptin and aprotinin). Membranes were obtained by two sequential centrifugations. Standard phosphorylation assays were performed with equivalent protein amounts $\left(10 \mathrm{~min}, 37^{\circ} \mathrm{C}\right)$. The reaction was terminated by adding $1 \mathrm{M} \mathrm{HCl}$, and lipids were extracted with $\mathrm{CHCl}_{3} / \mathrm{MeOH}(1: 1, \mathrm{v} / \mathrm{v})$. Organic layers were recovered, dried, dissolved in $20 \mu \mathrm{I} \mathrm{CHCl}_{3}$ and applied to silica gel TLC plates with dioleoyl-PA as a standard. Plates were developed in a $\mathrm{CHCl}_{3}, \mathrm{MeOH}, 4 \mathrm{M} \mathrm{NH}_{4} \mathrm{OH}$ solvent system (9:7:2, v/v/v), dried, autoradiographed and PA bands quantified by autoradiogram scanning.

Quantitative RT-PCR. Total RNA was reverse transcribed using the High Capacity cDNA Archive Kit (PN4322171; Applied Biosystems, Foster City, CA, USA). Real-time PCR reactions were performed in triplicate with Fluocycle II SYBR PCR Master Mix (ERD002250R; EuroClone, Pero, Italy) in 384-well optical plates. PCR reactions were run in an ABI PRISM 7900HT (Applied Biosystems) and SDS v.2.2 software was used to analyze the results by the comparative $\mathrm{Ct}$ method $(\Delta \Delta \mathrm{Ct})$. Primers used were: Runx1 - $5^{\prime}$-CCAGCAAGCTGAGGAGCG GCG-3'; 5'-CGGATTTGTAAAGACGGTGA-3'; Runx3 - 5'-TTCAACGACCTTCGA TTCGT-3'; 5'-TTGGTGAACACGGTGATTGT-3'; TOX - 5'-CTGGACCCCTACTAT TGCAAC- $3^{\prime} ; 5^{\prime}$-GCAGGCCATTGTGATTCATGG-3'; LEF-1 - 5'-TCCTGAAATCC CCACCTTC-3'; 5'-ACCCGTGATGGGATAAACAG-3'; ROR $\gamma$ - 5'-ACCTCTTTTCA CGGGAGGA-3'; $5^{\prime}$-TCCCACATCTCCCACATTG-3', TCF-1 - 5'-CAGCTCCCCCA TACTGTGAG-3'; $5^{\prime}$-TGCTGTCTATATCCGCAGGAA-3'; $18 \mathrm{~S}$ - $5^{\prime}$-GAGAGGGAA ATCGTGCGTGA-3'; $5^{\prime}$-ACATCTGCTGGAAGGTGGAC- $3^{\prime} ; \beta$-actin - $5^{\prime}$-GAGAGG GAAATCGTGCGTGA-3'; $5^{\prime}$-ACATCTGCTGGAAGGTGGAC-3'

Immunofluorescence. Cells were transferred to poly-L-lysine-coated chamber slides, fixed with $2 \%$ PFA and permeabilized with PBS $0.2 \%$ Triton $X-100$. Secondary antibodies were Cy3-anti-mouse immunoglobulin (Jackson Laboratories) and Alexa488-anti-rabbit immunoglobulin (Molecular Probes). Cells were imaged with a laser scanning Olympus Fluoview 1000 confocal microscope and images processed using ImageJ v.1.43 software (Bethesda, MD, USA).

Flow cytometry. Cells were harvested, washed and stained with antibodies in PBS staining buffer (with $2 \% \mathrm{FBS}$ and $0.02 \% \mathrm{NaN}_{3}$ ). The antibodies used for flow cytometric analysis, anti-rat CD2 (OX-34); anti-CD4 (6K1.5), anti-CD8 (53-5.8), anti-CD25 (7D4), anti-HSA (M1/69), anti-TCR $\beta$ (H57-597), anti-CD69 (H1.2F3), anti-CD44 (IM7), anti-CD25 (7D4), anti-TCR $\gamma \delta$ (GL3), anti-Ter119, anti-Gr1 (RB6-8C5) and anti-NK1.1 (PK136), were purchased from BD Pharmingen 
(Franklin Lakes, NJ, USA); anti-B220 (RA3-6B2) and anti-lgM (11E10) were from Beckman Coulter (Pasadena, CA, USA); anti-CD5 (B19.1) was from Southern (Surrey, UK); anti-CD3 (145-2C11) was from BD Biosciences (Franklin Lakes, NJ, USA); anti-FoxP3 (FJK-165) was from eBioscience (San Diego, CA, USA); and anti-human CD69 (TP1.55.3) was from Immunotech (Monrovia, CA, USA). CD1d tetramers (PBS57/unloaded Alexa488) were from NIH Tetramer Facility (Atlanta, GA, USA). Intracellular staining was performed with commercial buffers (Cytofix, BD Biosciences; Perm buffer III) for rat CD2, TCR $\beta$, phospho-ERK and FoxP3 detection. Samples were collected on a FACSCalibur (BD Biosciences) and analyzed with FlowJo software v.8.8.6 (Tree Star, Ashland, OR, USA). Thymus subpopulations were sorted on a BD FACSAria (BD cytometer, Franklin Lakes, NJ, USA).

\section{Conflict of Interest}

The authors declare no conflict of interest.

Acknowledgements. We thank IM group members for helpful discussion and $C$ Mark for excellent editorial assistance. MA receives a fellowship from the Madrid Regional Government and EA receives a FPU fellowship from the Spanish Ministry of Education. This work was supported in part by grants from the Spanish Ministry of Economy and Competitivity (BFU2010-21138), the Spanish Ministry of Health (RD120036/0059) and the Madrid regional government (S2010/BMD-2326) to IM.

\section{Author Contributions}

IM and MA designed the project, the experimental approach and wrote the manuscript; MA, EA and RL performed the experiments.

1. Goodnow CC, Sprent J, Fazekas de St Groth B, Vinuesa CG. Cellular and genetic mechanisms of self tolerance and autoimmunity. Nature 2005; 435: 590-597.

2. Carpenter AC, Bosselut R. Decision checkpoints in the thymus. Nat Immunol 11: 666-673.

3. Kruisbeek AM, Haks MC, Carleton M, Michie AM, Zuniga-Pflucker JC, Wiest DL. Branching out to gain control: how the pre-TCR is linked to multiple functions. Immunol Today 2000; 21: $637-644$.

4. Pennington DJ, Silva-Santos B, Hayday AC. Gammadelta T cell development - having the strength to get there. Curr Opin Immunol 2005; 17: 108-115.

5. Singer A, Adoro S, Park JH. Lineage fate and intense debate: myths, models and mechanisms of CD4- versus CD8-lineage choice. Nat Rev Immunol 2008; 8: 788-801.

6. Carrasco S, I Merida. Diacylglycerol when simplicity becomes complex. Trends Biochem Sci 2007; 32: 27-36

7. Tognon CE, Kirk HE, Passmore LA, Whitehead IP, Der CJ, Kay RJ. Regulation of RasGRP via a phorbol ester-responsive C1 domain. Mol Cell Biol 1998; 18: 6995-7008.

8. Stone JC. Regulation and function of the RasGRP family of Ras activators in blood cells. Genes Cancer 2011; 2: 320-334.

9. Li YQ, Hii CS, Costabile M, Goh D, Der CJ, Ferrante A. Regulation of lymphotoxin production by the p21ras-raf-MEK-ERK cascade in PHA/PMA-stimulated Jurkat cells. $\mathrm{J}$ Immunol 1999; 162: 3316-3320.

10. Merida I, Avila-Flores A, Merino E. Diacylglycerol kinases: at the hub of cell signalling. Biochem J 2008; 409: 1-18.

11. Sanjuan MA, Jones DR, Izquierdo M, Merida I. Role of diacylglycerol kinase alpha in the attenuation of receptor signaling. J Cell Biol 2001; 153: 207-220.

12. Olenchock BA, Guo R, Carpenter JH, Jordan M, Topham MK, Koretzky GA et al. Disruption of diacylglycerol metabolism impairs the induction of T cell anergy. Nat Immunol 2006; 7: 1174-1181.

13. Guo R, Wan CK, Carpenter JH, Mousallem T, Boustany RM, Kuan CT et al. Synergistic control of $\mathrm{T}$ cell development and tumor suppression by diacylglycerol kinase alpha and zeta. Proc Natl Acad Sci USA 2008; 105: 11909-11914.

14. Marklund U, Lightfoot K, Cantrell D. Intracellular location and cell context-dependent function of protein kinase D. Immunity 2003; 19: 491-501.

15. Daniels MA, Teixeiro E, Gill J, Hausmann B, Roubaty D, Holmberg K et al. Thymic selection threshold defined by compartmentalization of Ras/MAPK signalling. Nature 2006; 444 : 724-729.

16. Hay N, Sonenberg N. Upstream and downstream of mTOR. Genes Dev 2004; 18 : 1926-1945.

17. Gorentla BK, Wan CK, Zhong XP. Negative regulation of mTOR activation by diacylglycerol kinases. Blood 2011; 117: 4022-4031.

18. Hogquist KA. Signal strength in thymic selection and lineage commitment. Curr Opin Immunol 2001; 13: 225-231.
19. Norment AM, Bogatzki LY, Klinger M, Ojala EW, Bevan MJ, Kay RJ. Transgenic expression of RasGRP1 induces the maturation of double-negative thymocytes and enhances the production of CD8 single-positive thymocytes. J Immunol 2003; 170: 1141-1149.

20. Moran AE, Holzapfel KL, Xing Y, Cunningham NR, Maltzman JS, Punt $\mathrm{J}$ et al. T cell receptor signal strength in Treg and iNKT cell development demonstrated by a novel fluorescent reporter mouse. J Exp Med 2011; 208: 1279-1289.

21. Fu G, Chen Y, Yu M, Podd A, Schuman J, He Y et al. Phospholipase C\{gamma\}1 is essential for T cell development, activation, and tolerance. J Exp Med 2010; 207: 309-318.

22. Shen S, Zhu M, Lau J, Chuck M, Zhang W. The essential role of LAT in thymocyte development during transition from the double-positive to single-positive stage. J Immunol 2009; 182: 5596-5604.

23. Paterson DJ, Williams AF. An intermediate cell in thymocyte differentiation that expresses CD8 but not CD4 antigen. J Exp Med 1987; 166: 1603-1608.

24. Xiao SY, Li Y, Chen WF. Kinetics of thymocyte developmental process in fetal and neonatal mice. Cell Res 2003; 13: 265-273.

25. Yu Q, Erman B, Park JH, Feigenbaum L, Singer A. IL-7 receptor signals inhibit expression of transcription factors TCF-1, LEF-1, and RORgammat: impact on thymocyte development. J Exp Med 2004; 200: 797-803.

26. Telfer JC, Hedblom EE, Anderson MK, Laurent MN, Rothenberg EV. Localization of the domains in Runx transcription factors required for the repression of CD4 in thymocytes. J Immunol 2004; 172: 4359-4370.

27. Outram SV, Crompton T, Merida I, Varas A, Martinez AC. Diacylglycerol kinase alpha activity promotes survival of CD4 $+8+$ double positive cells during thymocyte development. Immunology 2002; 105: 391-398.

28. Anderson G, Hare KJ, Jenkinson EJ. Positive selection of thymocytes: the long and winding road. Immunol Today 1999; 20: 463-468.

29. Perez-Villar JJ, Whitney GS, Bowen MA, Hewgill DH, Aruffo AA, Kanner SB. CD5 negatively regulates the T-cell antigen receptor signal transduction pathway: involvement of SH2-containing phosphotyrosine phosphatase SHP-1. Mol Cell Biol 1999; 19: 2903-2912.

30. Chen Y, Ci X, Gorentla B, Sullivan SA, Stone JC, Zhang W et al. Differential requirement of RasGRP1 for gammadelta T cell development and activation. J Immunol 2012; 189: 61-71.

31. Almena M, Merida I. Shaping up the membrane: diacylglycerol coordinates spatial orientation of signaling. Trends Biochem Sci 2011; 36: 593-603.

32. Zhong XP, Hainey EA, Olenchock BA, Jordan MS, Maltzman JS, Nichols KE et al. Enhanced $T$ cell responses due to diacylglycerol kinase zeta deficiency. Nat Immunol 2003; 4: 882-890.

33. Kesavan KP, Isaacson CC, Ashendel CL, Geahlen RL, Harrison ML. Characterization of the in vivo sites of serine phosphorylation on Lck identifying serine 59 as a site of mitotic phosphorylation. J Biol Chem 2002; 277: 14666-14673.

34. Sharp LL, Schwarz DA, Bott CM, Marshall CJ, Hedrick SM. The influence of the MAPK pathway on T cell lineage commitment. Immunity 1997; 7: 609-618.

35. Shen S, Chen Y, Gorentla BK, Lu J, Stone JC, Zhong XP. Critical roles of RasGRP1 for invariant NKT cell development. J Immunol 2011; 187: 4467-4473.

36. Prince AL, Yin CC, Enos ME, Felices M, Berg LJ. The Tec kinases Itk and Rlk regulate conventional versus innate T-cell development. Immunol Rev 2009; 228: 115-131.

37. Kortum RL, Sommers CL, Alexander CP, Pinski JM, Li W, Grinberg A et al. Targeted Sos1 deletion reveals its critical role in early T-cell development. Proc Natl Acad Sci USA 2011; 108: $12407-12412$

38. Zha Y, Marks R, Ho AW, Peterson AC, Janardhan S, Brown I et al. T cell anergy is reversed by active Ras and is regulated by diacylglycerol kinase-alpha. Nat Immunol 2006; 7: $1166-1173$.

39. Martinez-Moreno M, Garcia-Lievana J, Soutar D, Torres-Ayuso P, Andrada E, Zhong XP et al. FoxO-dependent regulation of diacylglycerol kinase alpha gene expression. Mol Cell Biol 2012; 32: 4168-4180.

40. Xu M, Sharma A, Hossain MZ, Wiest DL, Sen JM. Sustained expression of pre-TCR induced beta-catenin in post-beta-selection thymocytes blocks $\mathrm{T}$ cell development. J Immunol 2009; 182: 759-765.

41. Haks MC, Belkowski SM, Ciofani M, Rhodes M, Lefebvre JM, Trop S et al. Low activation threshold as a mechanism for ligand-independent signaling in pre-T cells. J Immunol 2003; 170: 2853-2861.

42. Gallo EM, Winslow MM, Cante-Barrett K, Radermacher AN, Ho L, McGinnis L et al. Calcineurin sets the bandwidth for discrimination of signals during thymocyte development. Nature 2007; 450: 731-735.

43. Priatel JJ, Chen $X$, Dhanii S, Abraham N, Teh HS. RasGRP1 transmits prodifferentiation TCR signaling that is crucial for CD4 T cell development. J Immunol 2006; 177: 1470-1480.

44. Reif K, Burgering BM, Cantrell DA. Phosphatidylinositol 3-kinase links the interleukin-2 receptor to protein kinase B and p70 S6 kinase. J Biol Chem 1997; 272: 14426-14433.

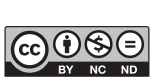

Cell Death and Disease is an open-access journal published by Nature Publishing Group. This work is licensed under a Creative Commons Attribution-NonCommercialNoDerivs 3.0 Unported License. To view a copy of this license, visit http://creativecommons.org/licenses/by-nc-nd/3.0/ 